\title{
Identification of a Strong Anthocyanin Activator, VbMYBA, From Berries of Vaccinium bracteatum Thunb.
}

\section{OPEN ACCESS}

Edited by:

Lourdes Gómez-Gómez,

University of Castilla-La Mancha,

Spain

Reviewed by:

Ivana Tomaz,

University of Zagreb, Croatia

Kaili Chen,

Northwest A\&F University, China

*Correspondence:

Zhi-Zhen Fang

Fzhzh2008@163.com

Specialty section:

This article was submitted to

Plant Metabolism

and Chemodiversity,

a section of the journal

Frontiers in Plant Science

Received: 04 May 2021 Accepted: 09 November 2021

Published: 06 December 2021

Citation:

Zhang Y-L, Lin-Wang K, Albert NW, Elborough C, Espley RV,

Andre CM and Fang Z-Z (2021)

Identification of a Strong Anthocyanin Activator, VbMYBA, From Berries of Vaccinium bracteatum Thunb.

Front. Plant Sci. 12:697212. doi: 10.3389/fpls.2021.697212

\section{Ya-Ling Zhang ${ }^{1}$, Kui Lin-Wang ${ }^{2}$, Nick W. Albert ${ }^{2}$, Caitlin Elborough ${ }^{2}$, Richard V. Espley ${ }^{2}$, Christelle M. Andre ${ }^{2}$ and Zhi-Zhen Fang ${ }^{1 *}$}

${ }^{1}$ Fruit Research Institute, Fujian Academy of Agricultural Sciences, Fuzhou, China, ${ }^{2}$ The New Zealand Institute for Plant and Food Research Limited, Mt Albert Research Centre, Auckland, New Zealand

Wufanshu (Vaccinium bracteatum Thunb.), which is a wild member of the genus Vaccinium, accumulates high concentration of anthocyanin in its berries. In this study, the accumulated anthocyanins and their derivatives in Wufanshu berries were identified through UHPLC-MS/MS analysis. Candidate anthocyanin biosynthetic genes were identified from the transcriptome of Wufanshu berries. qRT-PCR analyses showed that the expression of anthocyanin structural genes correlated with anthocyanin accumulation in berries. The R2R3-MYB, VbMYBA, which is a homolog of anthocyanin promoting R2R3-MYBs from other Vaccinium species, was also identified. Transient expression of VbMYBA in Nicotiana tabacum leaves confirmed its role as an anthocyanin regulator, and produced a higher anthocyanin concentration when compared with blueberry VcMYBA expression. Dual-luciferase assays further showed that VbMYBA can activate the DFR and UFGT promoters from other Vaccinium species. VbMYBA has an additional 23 aa at the $\mathrm{N}$ terminus compared with blueberry VcMYBA, but this was shown not to affect the ability to regulate anthocyanins. Taken together, our results provide important information on the molecular mechanisms responsible for the high anthocyanin content in Wufanshu berries.

Keywords: Vaccinium Bracteatum Thunb., anthocyanin biosynthesis, transcriptome, VbMYBA, R2R3 MYB

\footnotetext{
Abbreviations: 4CL, 4-coumaroyl:CoA-ligase; ANS, anthocyanin synthase; PAL, phenylalanine ammonia-lyase; C4H, cinnamate-4-hydroxylase; CHS, chalcone synthase; COG, Clusters of Orthologous Groups database; DAD, diode array detector; eggNOG, evolutionary genealogy of genes: Non-supervised Orthologous Groups database; ESI, electrospray ion; F3H, flavanone-3-hydroxylase; F3' $\mathrm{H}$, flavonoid3'-hydroxylase; F3' $5^{\prime} \mathrm{H}$, flavonoid $3^{\prime}, 5^{\prime}$-hydroxylase; FPKM, fragments per kilobase per million mapped reads; DFR, dihydroflavonol-4-reductase; GST, glutathione S-transferase; HPLC, high performance liquid chromatography; KEGG, Kyoto Encyclopedia of Genes and Genomes; KOG, euKaryotic Ortholog Groups of proteins database; MBW, MYB-bHLH-WD40; MS, mass spectrometry; Nr, NCBI non-redundant protein database; Pfam, Pfam-protein family database; qRT-PCR, real-time quantitative RT-PCR; RNA-Seq, RNA-sequencing; Swiss-Prot, Swiss-Prot protein database; UFGT, UDPglucose:flavonoid-3-O-glucosyltransferase; GO, Gene Ontology database; UHPLC, ultra-high performance chromatography system.
} 


\section{INTRODUCTION}

Wufanshu (Vaccinium bracteatum Thunb.) is a wild blueberry species belonging to the genus Vaccinium. It is an evergreen shrub or small tree widely distributed in hilly areas of China (Wang et al., 2013; Fan et al., 2019). In China, it is a traditional herbal medicine used to treat many diseases, such as inflammation, diarrhea, and skin eruptions (Wang et al., 2004; Fan et al., 2019; Zheng et al., 2019). Leaf juice of Wufanshu was used to stain and cook well-known local traditional food named $\mathrm{Wu}$ Fan in China (Zhang et al., 2014). Pigments derived from leaves of Wufanshu were also widely used to dye hair and clothing (Xu et al., 2017; Fan et al., 2020). Wufanshu leaves are rich in health-promoting compounds such as polysaccharides and flavonoids (Wang et al., 2013; Zhang et al., 2014; Fan et al., 2018). It has been reported that its extracts have health beneficial activities, such as tyrosinase inhibiting, anti-inflammatory, antifatigue, anti-diabetic and anti-cancer (Wang et al., 2010, 2013; Landa et al., 2014; Oh et al., 2018a,b; Fan et al., 2019). More particularly, the presence of secondary plant metabolites, such as anthocyanins (Lee et al., 2014), in Wufanshu berry extracts has been associated with its health-promoting activities such as antiproliferative, anti-inflammatory and antidepressant-like activity (Landa et al., 2014; Oh et al., 2018a). The amount of anthocyanins in fruits of Vaccinium species varies between species and cultivars (Lohachoompol et al., 2008; Stevenson and Scalzo, 2012; Li et al., 2017). Cho et al. (2012) showed that the anthocyanin concentration in berries of $V$. bracteatum Thunb. was higher than that of $V$. corymbosum, but the basis for this is unknown.

Anthocyanins belong to the flavonoid class of polyphenolics and are produced by a complex biosynthetic pathway. This starts with the condensation of three molecules of malonyl $\mathrm{CoA}$ and one molecule of 4-coumaroyl CoA by phenylalanine ammonia-lyase (PAL), cinnamate-4-hydroxylase $(\mathrm{C} 4 \mathrm{H})$, 4-coumaroyl:CoA-ligase (4CL), chalcone synthase (CHS). The naringenin chalcone formed from this is then converted to naringenin flavanone by chalcone isomerase $(\mathrm{CHI})$, and then hydroxylated by flavanone-3-hydroxylase (F3H), to form dihydrokaempferol. At this stage the pathway can branch to form different anthocyanins, such as cyanidin or delphinidin. Further hydroxyl groups are added at this stage by flavonoid $3^{\prime}$-hydroxylase $\left(\mathrm{F}^{\prime} \mathrm{H}\right)$ or flavonoid $3^{\prime}, 5^{\prime}$-hydroxylase $\left(\mathrm{F}^{\prime} 5^{\prime} \mathrm{H}\right)$ before conversion by dihydroflavonol-4-reductase (DFR). The formation of anthocyanidins (aglycones) is controlled by anthocyanidin synthase (ANS), and these are glycosylated by UDPglucose:flavonoid-3-O-glucosyltransferase (UFGT) to form anthocyanins (Holton and Cornish, 1995). Additional chemical modifications of the basic anthocyanin molecules with additional sugars, methyl and acyl groups can generate a large variety of anthocyanins that differ in color and chemical properties. Many of these biosynthetic steps are shared with proanthocyanidin (condensed tannin) biosynthesis, but the activity of UFGT is necessary for anthocyanin production. Thus, it is a key point of regulation in fruit species that have complex flavonoid profiles, such as grape and Vaccinium spp. (Kobayashi et al., 2002; Günther et al., 2020).
In Vaccinium species, such as blueberry, the most common anthocyanins are aglycones of cyanidin (cyanidin 3-galactoside, cyanidin 3-arabinoside and cyanidin 3-glucoside), peonidin (peonidin 3-glucoside, peonidin 3-galactoside and peonidin 3-arabinoside), delphinidin (delphinidin 3-galactoside, delphinidin 3-glucoside and delphinidin 3-arabinoside), malvidin (malvidin 3-arabinoside), and petunidin (petunidin 3 -galactoside, petunidin 3-arabinoside and petunidin 3glucoside) (Lohachoompol et al., 2008). The network that regulates anthocyanin biosynthesis in plants has been studied extensively (Jaakola, 2013). While all three components are necessary for anthocyanin regulation, the central component of the network that regulates anthocyanin biosynthesis is the MYB-bHLH-WD40 (MBW) complex (Nesi et al., 2001; Gonzalez et al., 2008; Albert et al., 2014) and R2R3-MYBs have been shown to be the key regulators across a range of plant species (Espley et al., 2007; Hichri et al., 2011; Albert et al., 2014; Feng et al., 2015; Tuan et al., 2015; Jin et al., 2016; Butelli et al., 2017; Plunkett et al., 2018; Zhang et al., 2018). This is because the MYB protein is usually the limiting component of the MBW complex, and because the MYB can activate the expression of bHLH and WDR genes through a widely conserved hierarchy (Albert et al., 2014, 2021). In Vaccinium species, R2R3-MYBs have been reported to be involved in anthocyanin accumulation (Nguyen et al., 2017; Plunkett et al., 2018; Die et al., 2020) and down regulation of their expression was associated with a reduction or loss of anthocyanin accumulation in berries (Primetta et al., 2015; Yang et al., 2018; Die et al., 2020). The regulators of anthocyanin biosynthesis of Wufanshu have not yet been identified. Understanding the regulatory mechanisms in these the highly anthocyanic berries will increase our knowledge of anthocyanin accumulation in the Vaccinium genus.

In this study, we report on the identification of $V b M Y B A$, which is closely related to the R2R3-MYB anthocyanin activator $V c M Y B A$ from blueberries ( $V$. corymbosum and $V$. virgatum) (Plunkett et al., 2018) and show that its anthocyanin promoting activity is stronger than VcMYBA. Our results will help the development of new Vaccinium cultivars that can accumulate more anthocyanins in their berries.

\section{MATERIALS AND METHODS}

\section{Plant Materials}

All berries at different stages were harvested from wild Wufanshu plants in Lianjiang, in the city of Fuzhou (Fujian Province, China). Green berries were harvested on June 19, 2016. Red and black berries were harvested on November 16, 2016 and divided into two groups according to color. We estimated that these berry stages approximately correspond with stages 5,6 , and 8 , described by for blueberry (Zifkin et al., 2011). Berries sampled from three different plants were used as biological replicates. For each plant, berries with similar size and color were pooled together. After harvesting, all samples were transported to lab and immediately frozen in liquid nitrogen and kept at $-80^{\circ} \mathrm{C}$ for further analysis. 


\section{Total Anthocyanin Content Analysis in Wufanshu Berries}

Approximately $1 \mathrm{~g}$ (FW) of whole berries was ground to fine powder in liquid nitrogen and extracted with $10 \mathrm{~mL}$ extraction solution $\left(0.05 \% \mathrm{HCl}\right.$ in methanol) at $4^{\circ} \mathrm{C}$ for $24 \mathrm{~h}$. Anthocyanin content was quantified using $\mathrm{pH}$ differential method as described previously (Fang et al., 2016).

\section{Anthocyanins Characterization of Wufanshu Berries}

Extraction, identification and quantification of metabolites were carried out at Suzhou BioNovoGene (Suzhou, China). Approximately $0.2 \mathrm{~g}(\mathrm{FW})$ of the berry powder was transferred to a $2 \mathrm{~mL}$ centrifuge tube containing $1 \mathrm{~mL}$ of water: methanol: formic acid (66.5: 28.5: 5, v/v/v), incubate on ice for $30 \mathrm{~min}$ and centrifuged at $18,756 \times g$ for $10 \mathrm{~min}$. The tube containing the mixture was centrifuged at $12,000 \mathrm{rpm}$ for $10 \mathrm{~min}$. The supernatants were freeze-dried and the extracts were resuspended with $0.2 \mathrm{~mL} 5 \%$ formic acid. An Ultra-high performance chromatography system (UHPLC, Vanquish, Thermo Fisher Scientific, Waltham, MA, United States) with a Waters HSS T3 $(2.1 \times 100 \mathrm{~mm}, 1.8 \mu \mathrm{m})$ column was used. The parameters used were as follows: flow rate of $300 \mu \mathrm{L} \mathrm{min}^{-1}$, column oven temperature of $40^{\circ} \mathrm{C}$, and sample size of $2 \mu \mathrm{L}$. The mobile phases were $0.1 \%$ formic acid in water $(\mathrm{A})$ and $0.1 \%$ formic acid in acetonitrile (B). The gradient elution procedure was as follows: 0-4.0 $\mathrm{min}, 10 \% \mathrm{~A} ; 4.0-12.0 \mathrm{~min}, 10-60 \% \mathrm{~A} ; 12.0-18.0 \mathrm{~min}$, $60 \%$ A constant; $18.0-18.1 \mathrm{~min}, 60-10 \%$ A; $18.1-26.0 \mathrm{~min}, 10 \%$ A constant. Mass spectrometry (MS) analysis was carried out on a Thermo Q Exactive instrument (Thermo Fisher Scientific, Waltham, MA, United States) equipped with an electrospray ion (ESI) source in positive mode. The capillary voltage was set to $3000 \mathrm{~V}$ and the capillary temperature was set to $320^{\circ} \mathrm{C}$. The pressure of sheath gas and auxiliary gas were set at 40 and 10 arbitrary units, respectively. Metabolites were detected using fullscan/MS2 mode with a resolution of 70000. ESI spectra were acquired through the information-dependent acquisition mode in Xcalibur 4.1 software (Thermo Fisher Scientific, Waltham, MA, United States). The dynamic exclusion time was set to 6s. For MS1, full MS spectra between 200 and 1500 mass-tocharge ratio $(\mathrm{m} / \mathrm{z})$ were recorded. MS/MS scans were recorded between 200 and $2000 \mathrm{~m} / \mathrm{z}$. Data dependent acquisition survey scans were acquired in $100 \mathrm{~ms}$ and the 10 most abundant product ion scans were collected. Each metabolite was confirmed based on their exact molecular weights, then the fragment information obtained according to the MS/MS mode was matched in database built by BioNovogene to identify metabolites. Searches were performed using the following settings: precursor ion $\mathrm{m} / \mathrm{z}$ tolerance: $\pm 10 \mathrm{ppm} ; \mathrm{MS} / \mathrm{MS} \mathrm{m} / \mathrm{z}$ tolerance: $\pm 20 \mathrm{ppm}$.

\section{RNA Extraction, cDNA Library Construction and Sequencing}

For RNA-Seq, berries at green or black stage from three replicates were mixed and subjected to RNA extraction. Total RNA extraction, cDNA library construction, and sequencing were performed by staff at Beijing BioMarker Technologies
(Beijing, China). RNA extraction was carried out using EZNA Plant RNA Kit (Omega Bio-tek) RNA purity was checked using the NanoPhotometer ${ }^{\circledR}$ spectrophotometer (IMPLEN, CA, United States). RNA concentration was measured using Qubit ${ }^{\circledR}$ RNA Assay Kit in Qubit 2.0 Fluorometer (Life Technologies, CA, United States). RNA integrity was assessed using the RNA Nano 6000 Assay Kit of the Agilent Bioanalyzer 2100 system (Agilent Technologies, CA, United States). cDNA library was generated using NEBNext ${ }^{\circledR}$ Ultra $^{\mathrm{TM}}$ RNA Library Prep Kit for Illumina ${ }^{\circledast}(\mathrm{NEB}$, United States) following manufacturer's recommendation. Library quality was assessed using the Agilent Bioanalyzer 2100 system. Sequencing of the cDNA libraries were carried out on the Illumina HiSeq ${ }^{\mathrm{TM}} \mathrm{X}$ Ten sequencing platform. The RNA-seq reads have been deposited in the NCBI Short Read Archive and are accessible under PRJNA694726.

\section{De novo Assembly, UniGene Annotation and Expression Quantification of Unigenes}

The raw reads were processed through in-house perl scripts and de novo assembled into unigenes with Trinity (Grabherr et al., 2011) as described previously (Zhang et al., 2018). Annotation of the assembled unigenes was performed using BLASTx $(E-v a l u e<10-5)$ searches against eight public databases including the NCBI non-redundant protein database (Nr), SwissProt protein database (Swiss-Prot), the Gene Ontology database (GO), the Clusters of Orthologous Groups database (COG), the euKaryotic Ortholog Groups of proteins database (KOG), the Kyoto Encyclopedia of Genes and Genomes (KEGG), the Pfamprotein family database (Pfam) and the evolutionary genealogy of genes: Non-supervised Orthologous Groups database (eggNOG). For calculation of gene expression level, clean RNA-Seq reads were mapped to the assembled unigenes using Bowtie (Langmead et al., 2009) and the expression levels of unigenes were calculated with fragments per kilobase per million mapped reads (FPKM) using RSEM (Li and Dewey, 2011).

\section{qRT-PCR (Real-Time Quantitative RT-PCR) Analysis}

Total RNA was extracted from green, red and black Wufanshu berries using EZNA Plant RNA Kit (Omega Bio-tek). First strand cDNA was prepared from $500 \mathrm{ng}$ total RNA HiScript III RT SuperMix for qPCR with gDNA wiper (Vazyme, Nanjing, China). qRT-PCR was performed using the Eppendorf Realplex ${ }^{4}$ realtime PCR system (Hamburg, Germany) in a total volume of $20 \mu \mathrm{L}$ in each well containing $10 \mu \mathrm{L}$ of $2 \times$ ChamQ Universal SYBR qPCR Master Mix (Vazyme, Nanjing, China), $6 \mu \mathrm{L}$ of cDNA (in 1:30 dilution), and $0.4 \mu \mathrm{L} 10 \mu \mathrm{M}$ primers. qRT-PCR conditions were $30 \mathrm{~s}$ at $95^{\circ} \mathrm{C}$, followed by 40 cycles of $5 \mathrm{~s}$ at $95^{\circ} \mathrm{C}, 15 \mathrm{~s}$ at $60^{\circ} \mathrm{C}$, followed by $60^{\circ} \mathrm{C}$ to $95^{\circ} \mathrm{C}$ melting curve detection. Actin gene (c87909.graph_c0) was used as the reference. The expression levels were calculated using the $2^{-\Delta \Delta C T}$ method. Three biological and four technical replications were performed. Primers for qRT-PCR were listed in Supplementary Table $\mathbf{1}$. Linear regression analysis of FPKM and qPCR was performed using Minitab ${ }^{\circledast} 18$. 


\section{Cloning of $V b M Y B A$ and Prediction of Amino Acid Sequence}

cDNA of black Wufanshu berries was generated using a First-Strand cDNA synthesis kit (Fermentas, United States) and used as template for gene cloning. The cDNA sequence encoding VbMYBA was isolated using $\mathrm{I}-5^{\mathrm{TM}} 2 \times$ HighFidelity Master Mix (MCLAB, San Francisco, CA) with primers (forward 5'-GGCAGCTTACATGAAAATTCTCC-3' and reverse 5'-CAAACAAAGAAATGCTTGCCG-3') designed according to unigene sequence. The generated PCR products were purified and subsequently cloned into the pEASY-Blunt Zero vector using pEASY-Blunt Zero Cloning Kit (TransGen, Beijing, China) and sequenced. The open-reading frame of the sequence was predicted using GenBank ORF finder ${ }^{1}$.

\section{Multiple Sequence Alignment and Phylogenetic Analysis}

Multiple sequence alignment of R2R3 MYB amino acid sequences from Wufanshu, blueberry, kiwifruit and peach was performed by CLUSTALW ${ }^{2}$. Shading of the alignment results was performed using ESPript 3.0 (Robert and Gouet, 2014). Phylogenetic tree was constructed using the maximum likelihood method with 1000 bootstrap replicates by MEGA-X. R2R3 MYB amino acid sequences used for phylogenetic analysis include Arabidopsis thaliana AtMYB4 (At4G38620), AtMYB11 (AT3G62610), AtMYB12 (AT2G47460), AtMYB75 (AT1G56650), AtMYB90 (AT1G66390), AtMYB111 (AT5G49330), AtMYB113 (AT1G66370), AtMYB114 (AT1G66380), AtMYB123 (AT5G35550); Actinidia chinensis AcMYB10 (PSS35990), and AcMYB110 (AHY00342); Fragaria $\times$ ananassa FaMYB1 (AAK84064.1), FaMYB9 (JQ989281), FaMYB10 (ABX79947.1); Gossypium hirsutum GhMYB1 (AAA33067.1), GhMYB6 (AAN28286.1) GhMYB10 (ABR01222.1), and GhMYB36 (AF336284); Lotus japonicus LjMYB12 (AB334529); Myrciaria cauliflora McMYB (MH383068); Malus $\times$ domestica MdMYB1 (XP_028963316.1), MdMYB9 (DQ267900), $\quad$ MdMYB10 (EU518249), MdMYB11 (DQ074463); Medicago truncatula MtLAP1 (ACN79541.1), and MtMYB14 (XP_003594801.1); Prunus cerasifera PcMYB10.1 (KP772281) and PcMYB10.2 (KP772282); Petunia hybrida PhMYB27 (AHX24372), PhAN2 (AB982128), PhDEEP PURPLE (ADQ00393.1), PhPURPLE HAZE (ADQ00388.1) and PhMYB4 (ADX33331.1); Prunus persica PpMYB10.1 (XM_007216468), PpMYB10.2 (XM_007216161) and PpMYB18 (KT159234); Prunus salicina PsMYB10.1 (MK105923), PsMYB10.2 (MK340932), and PsMYB18 (MK284223); PtMYB14 (ACR83705.1); Pyrus pyrifolia PyMYB10 (GU253310); Solanum lycopersicum SlMYB12 (ACB46530.1); Trifolium arvense TaMYB14 (AFJ53053.1); Trifolium repens TrMYB4 AMB27079), TrMYB133 (AMB27081), and TrMYB134 (AMB27082); Vaccinium ashei VaMYB (QOQ50851.1), Vaccinium corymbosum VcMYBA (MH105054); Vaccinium uliginosum VuMYBC2; Vitis vinifera VvMYBA2 (BAD18978), VvMYBF1 (ACV81697),

${ }^{1}$ https://www.ncbi.nlm.nih.gov/orffinder/

${ }^{2}$ https://www.genome.jp/tools-bin/clustalw
VvMYBC2-L1 (AFX64995.1), VvMYB-L2 (ACX50288.2), and VvMYBPA2 (ACK56131.1).

\section{Vector Construction}

Full-length coding sequence of $V b M Y B A$ was isolated using $2 \times$ Phanta Max Master Mix (Vazyme, Nanjing, China) with primer VbMYBAOEF and VbMYBAOER and inserted into pSAK277. A fragment (70-855 bp) of VbMYBA was amplified by primer VbMYB-NdelOEF and VbMYB-NdelOER. Fusion PCR was carried out to generate VcMYBA-Nadd (VcMYBA with 169 bp of $V b M Y B A$ ) were constructed as following: the $\mathrm{N}$-terminal of VbMYBA (1-69 bp) was amplified by primer VbMYBNF and VbMYBNR, and the VcMYBA contains overlap with $\mathrm{N}$-terminal of VbMYBA (1-69 bp) was amplified by VcMYBF and VcMYBR. Then the two fragments were fused through PCR. Finally, the VcMYBA-Nadd was amplified by primer VbMYB-NaddOEF and VbMYB-NaddOER, and inserted into pSAK277. The insertion of PCR fragments into pSAK277 was conducted using ClonExpress Ultra One Step Cloning Kit (Vazyme, Nanjing, China). Primer sequences used for the vector construction are listed in Supplementary Table 2. The promoter of $V c D F R$ was previously isolated (Plunkett et al., 2018) and the promoter of VavUFGT was isolated from Vaccinium virgatum using the primers VavpUFGTF (CTCCACATTTTTAACCTGGTGCAC) and VavpUFGT-R (CATGGTTATATTTTTGGTGGT), and cloned into pGreenII 0800-LUC (Hellens et al., 2005).

\section{Transient Transformation in Tobacco Leaf and Quantification of Anthocyanins}

Transient color assays were carried out in young leaves of Nicotiana tabacum seedlings grown in the greenhouse as described previously (Fang et al., 2021). All the constructs were transformed into Agrobacterium tumefaciens strain GV3101, and incubated at $28^{\circ} \mathrm{C}$ for 2 days. Agrobacterium cultures carrying constructs were resuspended in infiltration buffer containing $10 \mathrm{mM} \mathrm{MgCl} 2$ and $100 \mu \mathrm{M}$ acetosyringone $(\mathrm{pH}=5.7)$ and incubated at room temperature without shaking for $2 \mathrm{~h}$ before infiltration. Mixed bacterial cultures were injected into the abaxial side of young leaves. Many studies have suggested that basic helix-loop-helix (bHLH) transcription factors are indispensable partners of R2R3-MYBs (Espley et al., 2007; Gonzalez et al., 2008), in particular those belonging to the bHLH2 subgroup (PhAN1/AtTT8). Tobacco leaves express WDR and bHLH1 (PhJAF13/AtEGL3) orthologs, but require a bHLH2 (PhAn1/AtTT8) protein to fully regulate anthocyanin biosynthesis (Montefiori et al., 2015). Because the ability for the MYB to regulate the endogenous tobacco $b H L H 2$ gene is variable in transient assays, a bHLH2 (PsbHLH3) gene was co-transformed. We failed to clone the anthocyanin-associated bHLH from Wufanshu, and so we chose the plum anthocyaninactivating PsbHLH3 for transient color assays in tobacco leaves (Fang et al., 2021). This is a close homolog of peach PpbHLH3, which showed stronger anthocyanin-promoting activity than the homologous apple MdbHLH3 when coinfiltrated with blueberry VcMYBA (Plunkett et al., 2018). Separate strains containing VbMYBA and PsbHLH3 fused to the $35 \mathrm{~S}$ promoter in the 
pSAK277 vector and empty pSAK277 vector were infiltrated or co-infiltrated into the abaxial leaf surface. Blueberry $V c M Y B A$ (Plunkett et al., 2018) co-infiltrated with PsbHLH3 was used as positive control. Each infiltration was performed using three leaves from the same plants. Photographs were taken seven days after infiltration.

For quantification of anthocyanins, $10 \mathrm{mg}$ of freeze-dried tobacco leaves from the infiltrated area was mixed in $1 \mathrm{~mL}$ of methanol: water: formic acid (80: 19.5: 0.5, v/v/v) and shaken for $4 \mathrm{~h}$ at room temperature. The tube containing the mixture was centrifuged at $10,000 \times g$ for $15 \mathrm{~min}$. The supernatants were filtered through a $0.45 \mu \mathrm{m}$ PTFE syringe filter and submitted to high performance liquid chromatography (HPLC) analysis according to a method reported by Andre et al. (2012) with a few modifications. Briefly, quantification of the anthocyanins was performed using a Dionex Ultimate 3000 system (Sunnyvale, CA, United States) equipped with a diode array detector (DAD). A $5 \mu \mathrm{L}$ aliquot was injected onto a Thermo C18 Acclaim PolarAdvantage II column $(150 \times 2.1 \mathrm{~mm}$ i.d.; $3 \mu \mathrm{m}$ particle size $)$ (Waltham, MA, United States). The mobile phases were (A) $\mathrm{H}_{2} \mathrm{O}$ with $5 \%$ formic acid and (B) MeCN with $0.1 \%$ formic acid. The flow rate was $0.35 \mathrm{~mL} \mathrm{~min}{ }^{-1}$, and the column temperature was $35^{\circ} \mathrm{C}$. The $28 \mathrm{~min}$ gradient was as follows: $0-5 \mathrm{~min}, 7 \% \mathrm{~B}$ constant; 5-10 $\min , 7-12 \% \mathrm{~B}$; 10-20 $\mathrm{min}, 12-25 \% \mathrm{~B} ; 20-21 \mathrm{~min}, 25-100 \%$ B; 21-24 min, $100 \%$ B constant; $24 \mathrm{~min}, 7 \% \mathrm{~B} ; 24-28 \mathrm{~min}$, $7 \%$ B re-equilibration time. Monitoring was set at $520 \mathrm{~nm}$ for quantification. Anthocyanins were identified by their spectral data and were quantified as cyanidin-3-glucoside using fivepoint calibration curves. A validation standard was injected after every 10 th injection.

\section{Dual-Luciferase Assay}

Dual-luciferase assays were conducted in Nicotiana benthamiana leaves as reported previously (Lin-Wang et al., 2010). All the promoter constructs were individually transformed into Agrobacterium strain GV3101 that contains the pSoup plasmid using the electroporation method. Agrobacteria cultivation and infiltration preparation were performed according to the same protocol as described above for the transient color assay.

\section{RESULTS}

\section{Accumulation of Anthocyanin in Berries of Wufanshu}

The skin of unripe Wufanshu berries was initially green, then turned red and finally black when fully ripen (Figure 1A). The total content in anthocyanins increased during the ripening of berries and reached $516.97 \mathrm{mg} / 100 \mathrm{~g} \mathrm{FW}$ at the full ripe stage (Figure 1B). Individual anthocyanin compounds were characterized in ripe Wufanshu berries using UHPLC-MS/MS analysis. Delphinidin, cyanidin, malvidin, peonidin, petunidin, as well as pelargonidin derivatives could be identified based on their mass spectral data. Delphinidin 3-galactoside and 3-glucoside predominated the anthocyanin profile (Supplementary Table 3).

\section{RNA Sequencing and de novo Assembly of Wufanshu Berry Transcriptomes}

To identify genes responsible for anthocyanin accumulation in the berries of Wufanshu and investigate the underlying molecular mechanisms, transcriptomes of berries at green and black stages were generated by RNA-Seq. A total of $13.49 \mathrm{~Gb}$ clean reads were generated (Supplementary Table 4) and de novo-assembled into 87,811 unigenes. The average length of unigenes was $672.45 \mathrm{nt}$. Over one-half of the unigenes (61.59\%) were shorter than $500 \mathrm{bp}$, and only $18.59 \%(16,319)$ of the unigenes were longer than $1 \mathrm{~kb}$ (Supplementary Table 5).

All unigenes were annotated against eight public protein databases; NR, Swiss-Prot, Pfam, GO, COG, KOG, eggNOG4.5 and KEGG. In total, $49.37 \%(43,355)$ of the unigenes showed significant BLAST matches to known sequences in these databases (Supplementary Table 6). According to the
A

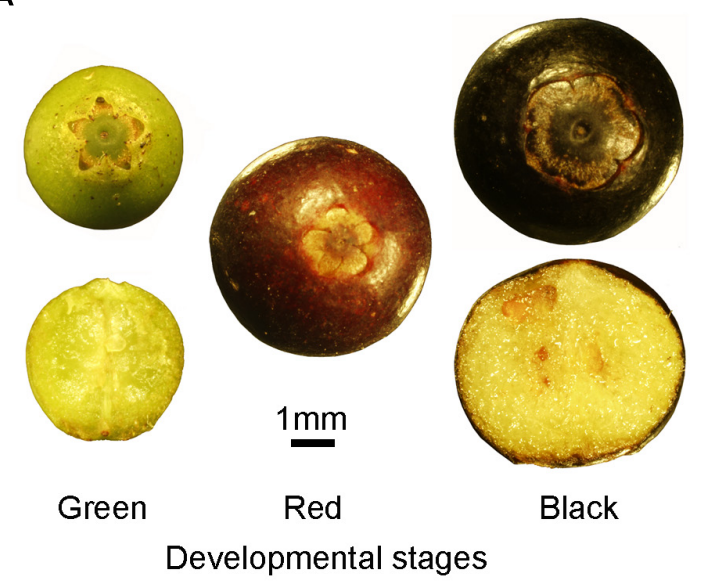

B

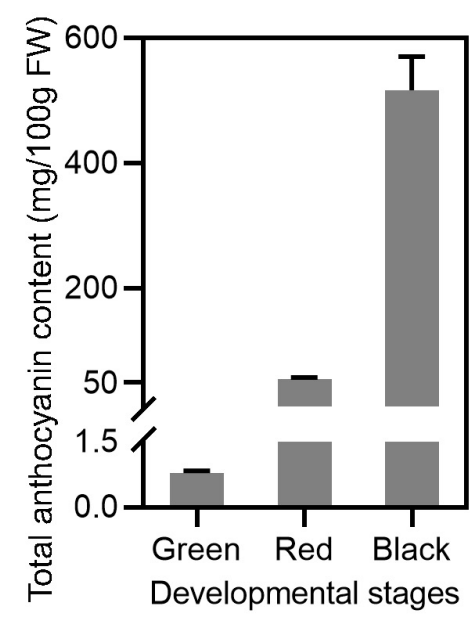

FIGURE 1 | Anthocyanin accumulation in the fruits of Wufanshu. (A) Fruits of Wufanshu at different stages. (B) Anthocyanin content in fruits of Wufanshu at different ripening stages. Error bars represent standard error of three replicates. Error bars represent standard error of five replicates. 
KEGG enrichment analysis, 168 unigenes were assigned to phenylpropanoid biosynthesis pathway (ko00940), 48 unigenes were assigned to flavonoid biosynthesis pathway (ko00941) and fourteen genes were involved in the anthocyanin biosynthesis pathway (ko00942) (Supplementary Table 7). Twenty-seven of these genes, which were more abundant in transcript levels in black Wufanshu berries (Supplementary Table 8), were identified as candidate genes involved in anthocyanin biosynthesis. c81310.graph_c0 was annotated as leucoanthocyanidin dioxygenase according to Swissprot database and c84705.graph_c0 was annotated as UDP-glucose: flavonoid 3-O-glucosyltransferase according to $\mathrm{Nr}$ database (Supplementary Table 8). In addition, c72505.graph_c0 was predicted to encode a homolog of cyclamen CkmGST3, reported to be involved in anthocyanin accumulation (Kitamura et al., 2012).

\section{Anthocyanin Biosynthetic Genes Are Upregulated in the Berries of Wufanshu}

Based on the RNA-Seq data, the expression levels of 11 anthocyanin biosynthetic genes with high FPKM values (maximum FPKM > 40) were analyzed by qRT-PCR. Our results indicated that the expression of $V b P A L$ (phenylalanine ammonia-lyase, c84812.graph_c0), Vb4CL (4-coumaroyl:CoA-ligase, c83421.graph_c0), VbC4H (cinnamate4-hydroxylase, c88472.graph_c0), VbCHS (chalcone synthase, c82998.graph_c0), $\quad \mathrm{VbF3H}$ (flavanone-3-hydroxylase, c85319.graph_c0 and c78308.graph_c0), VbF3'H (flavonoid3' hydroxylase, c88140.graph_c0), $V b F 3^{\prime} 5^{\prime} H$ (c94417.graph_c2), VbDFR (dihydroflavonol-4-reductase, c76847.graph_c0), VbUFGT (UDPglucose:flavonoid-3-O-glucosyltransferase, c84705.graph_c0), and VbGST (glutathione S-transferase, c81305.graph_c0) were upregulated during ripening of Wufanshu berries (Figures $\mathbf{2 A - K}$ ). Linear regression analysis showed that qRT-PCR value of the analyzed anthocyanin biosynthetic genes was significantly correlated with that FPKM value of them (Figure 2L).

\section{Identification of VbMYBA From Wufanshu Berries}

To identify candidate anthocyanin MYB activators, amino acid sequence of VcMYBA (Plunkett et al., 2018) from blueberry was
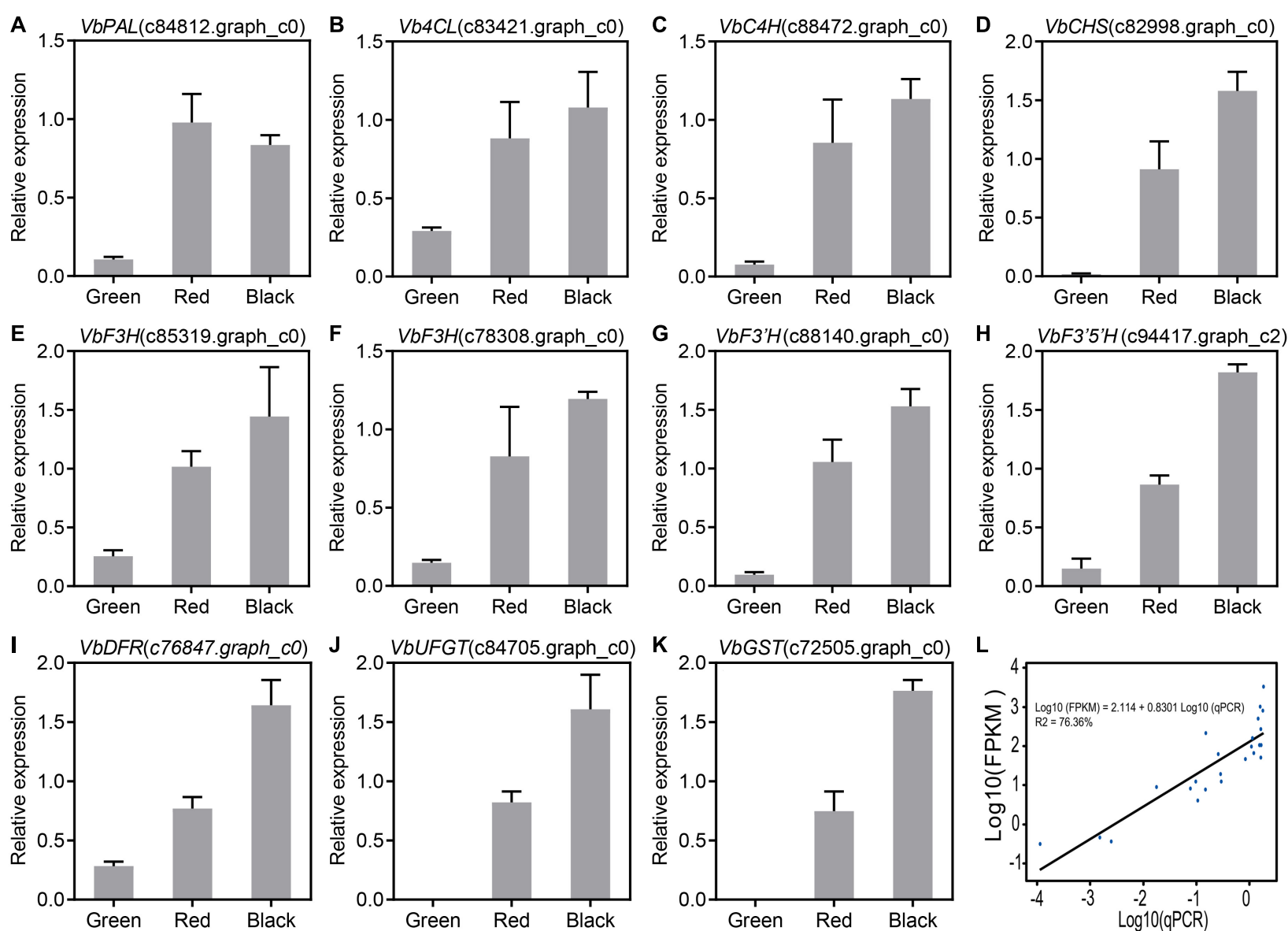

FIGURE 2 | Expression of candidate genes involved in anthocyanin biosynthesis. (A-K) qRT-PCR analysis of candidate genes involved in anthocyanin biosynthesis. Actin was used as the reference gene. The error bars represent the standard error of three biological replicates. (L) Linear regression analysis of gene expression levels obtained from RNA-Seq and quantitative real-time PCR data. 
used as query to BLAST against the assembled unigene sequences using TBtools (Chen et al., 2020). The BLAST analysis enabled the identification of a unigene (c67315.graph_c0) encoding MYB protein. The full-length open-reading frame of c67315.graph_c0 was amplified from cDNA of Wufanshu berries and confirmed by sequencing. The gene was designated as VbMYBA (GenBank Accession No. MW543447) (Supplementary Figure 1). BLASTp search against NCBI non-redundant protein sequences showed that VbMYBA protein have highest matching score to VcMYB1 (AYC35399.1) from $V$. corymbosum. Sequence alignment analysis indicated that VbMYBA protein showed high sequence identity to blueberry VcMYB1 and VcMYBA and contains conserved R2R3 domain and SG6 motif for anthocyanin-promoting MYBs (Figure 3). Nineteen amino acids, three in each $\mathrm{R}$ domain and 12 in C-terminal, were different between VbMYBA and VcMYBA (Figure 3). In addition, the N-terminal of VbMYBA protein was 6 aa and 23 aa longer than blueberry VcMYB1 and VcMYBA, respectively (Figure 3). Phylogenetic analysis showed that VbMYBA belongs to SG6 clade, which contains anthocyanin-activating MYB proteins from other plant species and is closely related to the anthocyanin-activators from Vaccinium species (Figure 4).

\section{VbMYBA Is a Strong Anthocyanin Activator}

Multiple sequences alignment and phylogenetic analysis suggested that VbMYBA was an anthocyanin-activator, similar to VcMYBA. RNA-Seq data along with qRT-PCR analysis indicated that the expression of $V b M Y B A$ was closely associated with that of anthocyanin biosynthetic genes and anthocyanin accumulation (Figure 5A). The function of VbMYBA was verified using tobacco leaf transient expression assays. Our results indicated that infiltration of $V b M Y B A$ with or without $\mathrm{Ps} b \mathrm{HLH} 3$ resulted in strong red pigmentation at infiltration sites 5 days after transformation, but only faint

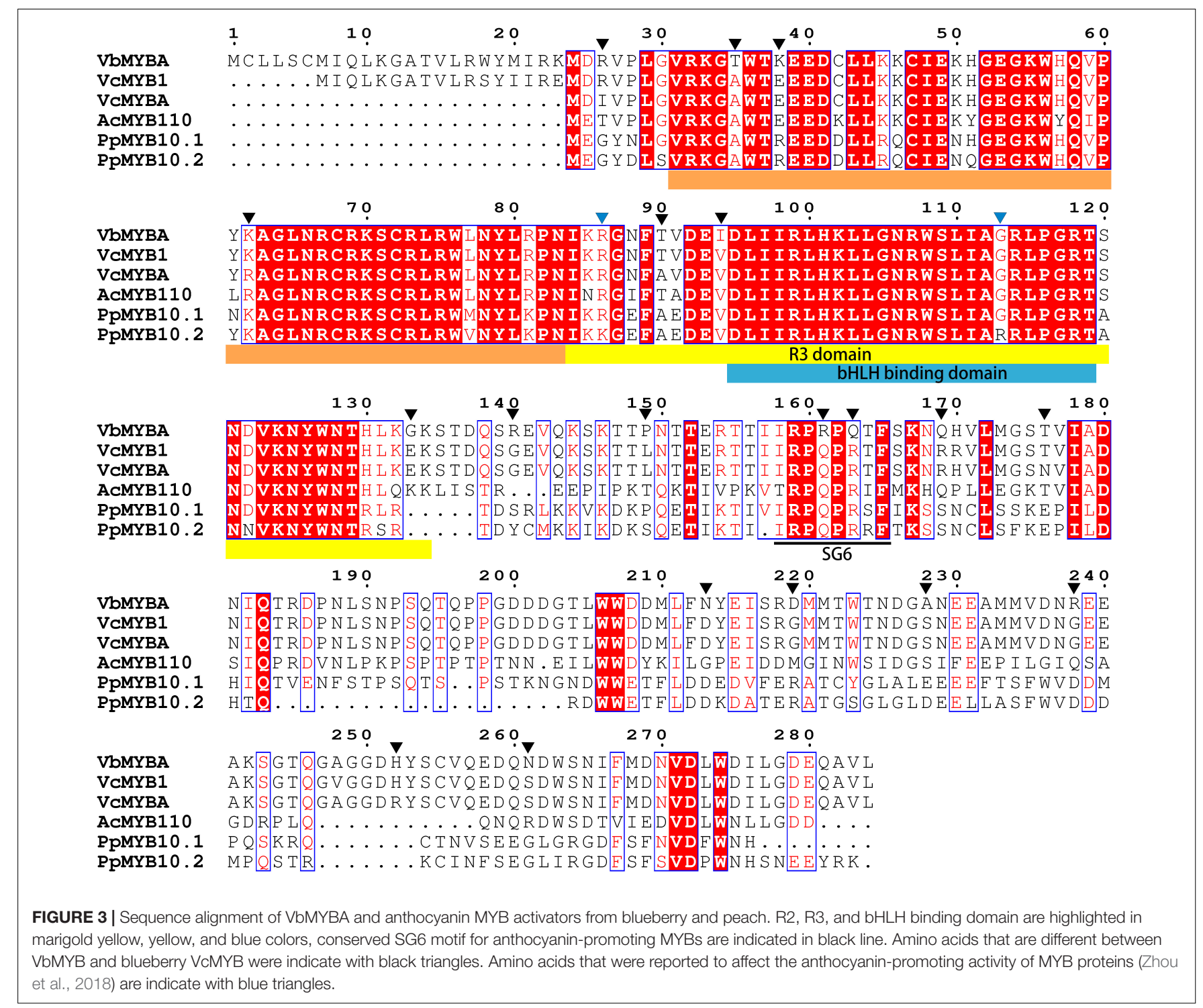




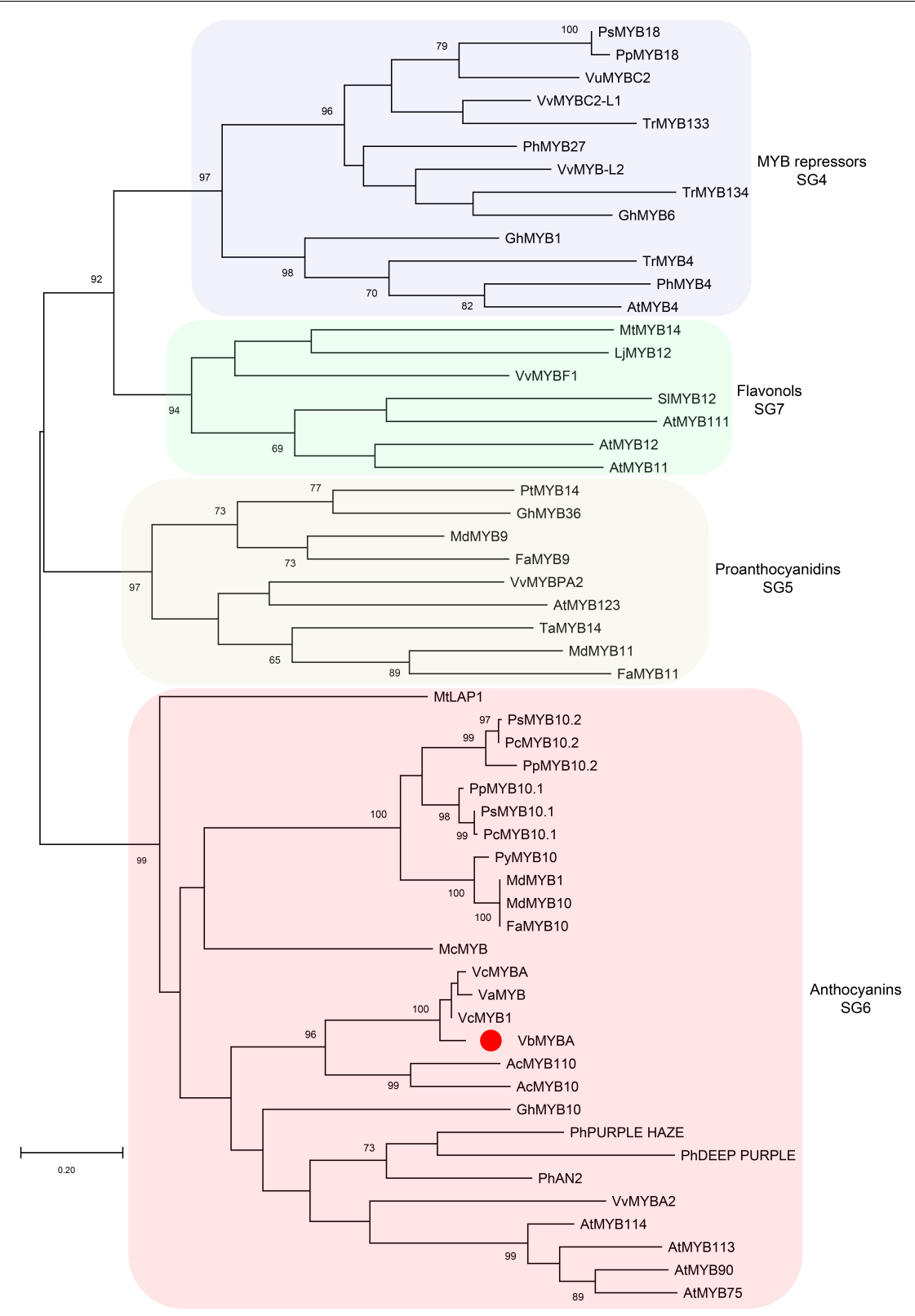

FIGURE 4 | Phylogenetic analysis of VbMYBA and R2R3 MYBs from other plant species. Phylogenetic tree was constructed using maximum-likelihood method by MEGA-X software. VbMYB from Wufanshu fruits is indicated with red dot. Phylogenetic groups are highlighted with different color shades. Numbers indicate node support $>65 \%$ from 1000 bootstrap replicates. The scale bar represents 0.2 substitutions per site.

red coloration was found when VcMYBA and PsbHLH3 were co-infiltrated (Figure 5B). Anthocyanin content analysis in leaves of N. tabacum indicated that no anthocyanin was detected in the infiltrated areas transformed with empty vector or PsbHLH3 (Figure 5C). A high concentration of anthocyanin was detected in tobacco leaves infiltrated with VbMYBA, while co-infiltration of VbMYBA and PsbHLH3 did not result in stronger anthocyanin accumulation in tobacco leaves (Figure 5C). However, the anthocyanin content in tobacco leaves infiltrated with $V c M Y B A$ and $P s b H L H 3$ was significantly lower than that in tobacco leaves infiltrated with $V b M Y B A$ with or without PsbHLH3 (Figure 5C). Among the anthocyanins detected in tobacco leaves, anthocyanin 1 and 2, which were likely correspond to a cyanidin-3-rutinoside and delphinidin3-rutinoside, respectively, according to their UV spectra and literature data, were the predominant forms (Figure 5C). In addition, we employed dual-luciferase assays to investigate the function of VbMYBA. The promoters of VcDFR and VavUFGT from blueberry (V. corymbosum and $V$. virgatum, respectively) were fused to a luciferase reporter. Infiltration of $V b M Y B A$ led to strong activation of promoters $V c D F R$ and $\operatorname{Vav} U F G T$ (Figures 5D,E). These results demonstrate that VbMYBA is a 

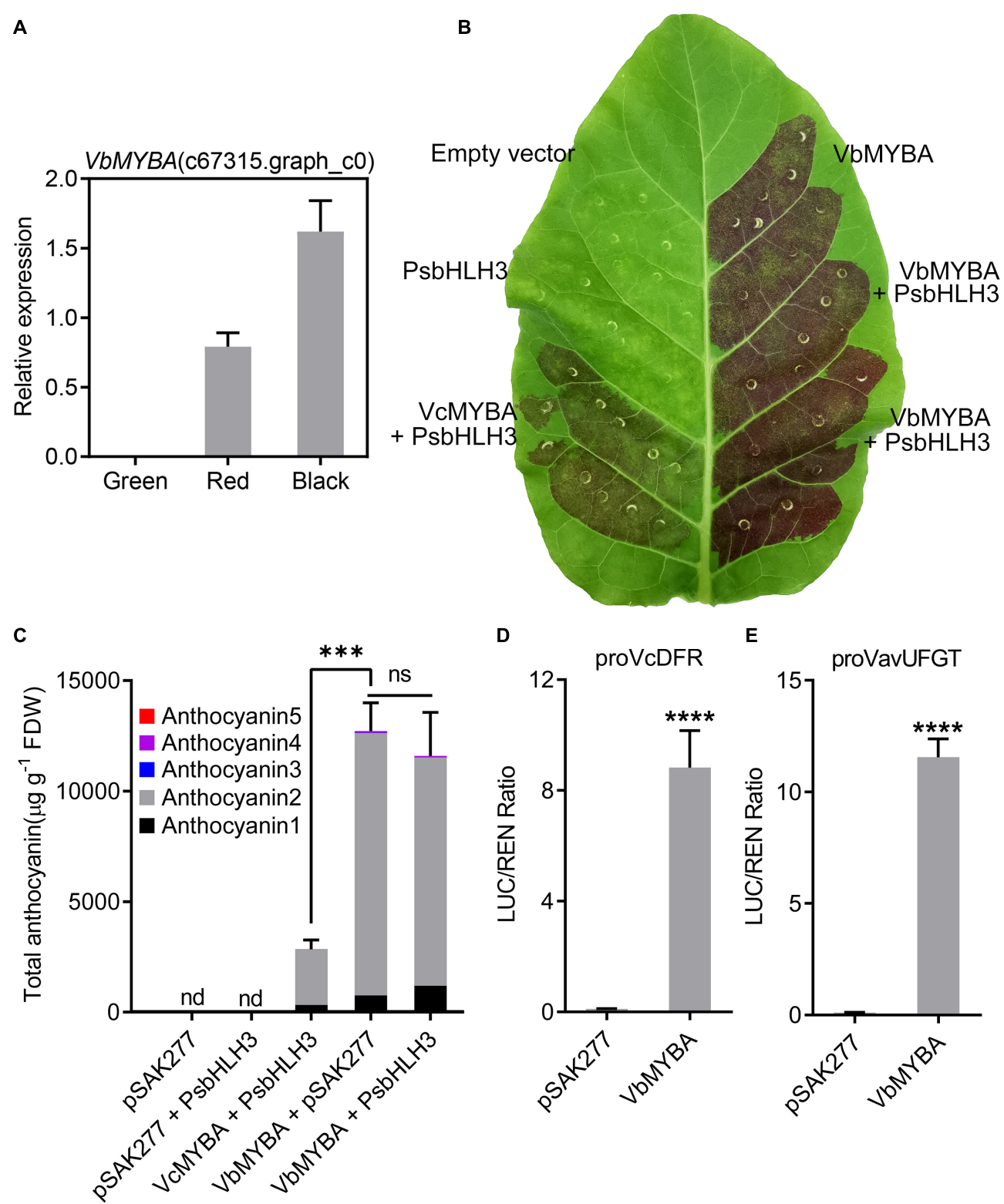

FIGURE 5 | Functional analysis of the VbMYBA gene using transient assay in tobacco. (A) Relative gene expression of $V b M Y B A$ in the fruits of Wufanshu. (B) Transient expression of VbMYBA in tobacco leaf. The photo was taken 5 days after infiltration. Blueberry VcMYBA/PsbHLH3 was used as positive control. (C) Anthocyanin content in transformed leaves of tobacco. Anthocyanins were measured as cyanidin-3-galactoside equivalents. Each value represents the mean of three biological replicates. FDW, freeze dry weight. nd, non-detected. Asterisks $\left.{ }^{\star \star \star}\right)$ indicate significant differences among treatments according to $t$-test $(P<0.0002)$. ns, non-significant. Analysis of the interaction of the VbMYBA gene with the promoters of VcDFR gene (D) from blueberry and VavUFGT gene (E) from $V$. virgatum using dual-luciferase reporter assay Nicotiana benthamiana leaves. Error bars represent the SEs for three replicates. Asterisks ( ${ }^{\star \star \star \star}$ ) indicate significant differences among treatments according to $t$-test $(P<0.0001)$.

strong anthocyanin activator and is capable of activating the promoters of DFR and UFGT from blueberry.

\section{N-Terminal Amino Acid Residues 1-23 of VbMYBA Is Not Responsible for Its Higher Anthocyanin-Promoting Activity}

Since the sequence for VbMYBA contained a 23 amino acid-residue insertion in the $\mathrm{N}$-terminal region compared with VcMYBA, we hypothesized that the N-terminal amino acid residues 1-23 of $\mathrm{VbMYBA}\left(\mathrm{N}^{1-23}\right)$ are responsible for the capacity for higher anthocyanin-promoting activity. To investigate whether the $\mathrm{N}^{1-23}$ is responsible for the divergence of anthocyanin-promoting activity between VbMYBA and VcMYBA, two mutant constructs were generated by deleting the $\mathrm{N}^{1-23}$ in VbMYBA (VbMYBA-Ndel) and fusing the $\mathrm{N}^{1-23}$ to VcMYBA (VcMYBA-Nadd) (Figure 6A). Anthocyanin-promoting activities of the mutant constructs 

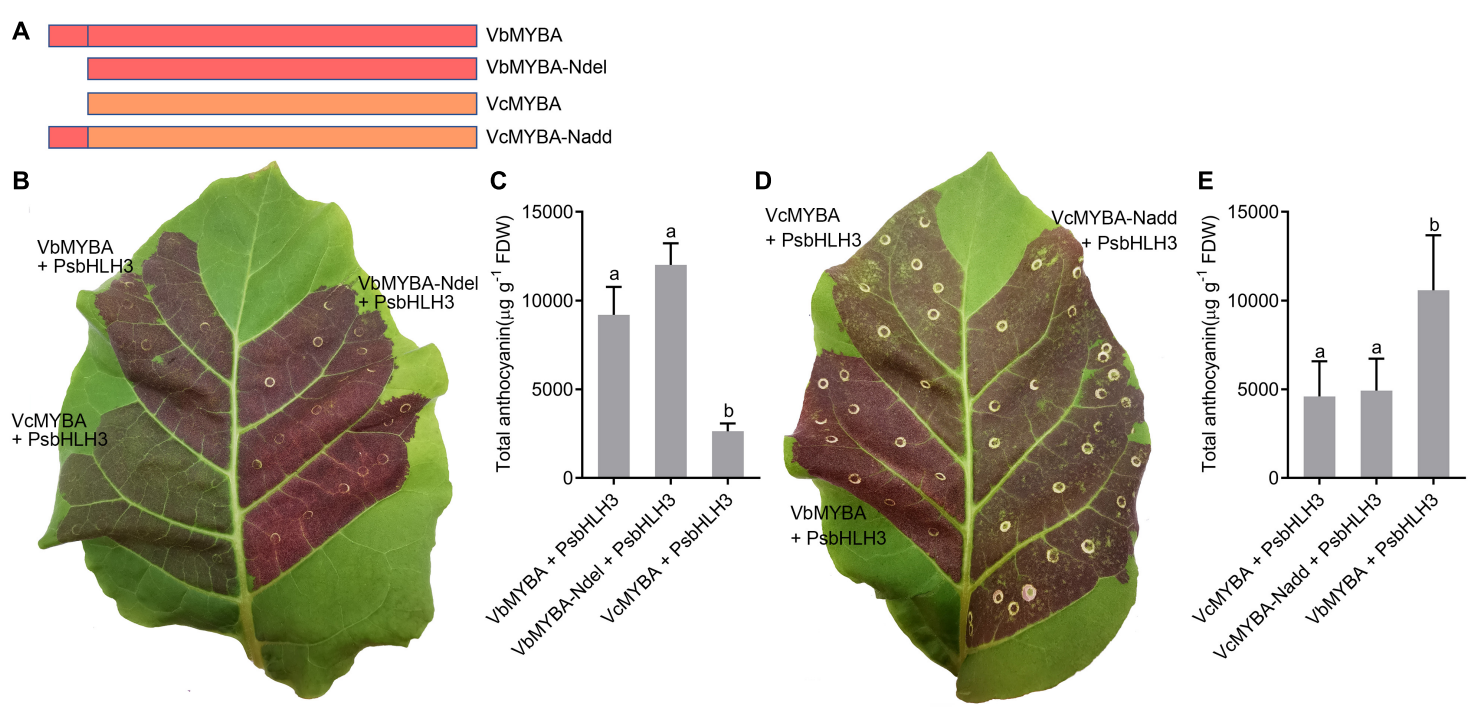

FIGURE 6 | Effect of N-terminal amino acid residues 1-23 of VbMYBA on anthocyanin-promoting activity. (A) Schematic diagram of mutants of VbMYBA and VcMYBA. (B,D) Transient expression of VbMYBA, VCMYBA and their mutants in tobacco leaf. The photo was taken 7 days after infiltration. (C,E) Anthocyanin content in transformed tobacco leaves. Error bars represent the standard errors for three replicates. Different lowercase letters indicate significant differences among treatments according to one-way ANOVA $(P<0.01)$.

were tested by tobacco leaf transient expression assays. Our results indicated that deletion of $\mathrm{N}^{1-23}$ in VbMYBA or insertion of $\mathrm{N}^{1-23}$ to VcMYBA have no significant effects on anthocyanin content in infiltrated tobacco leaves (Figures 6B-E). These results demonstrated that the N-terminal amino acid residues 1-23 of VbMYBA was not responsible for the divergence of anthocyanin-promoting activity between VbMYBA and VcMYBA.

\section{DISCUSSION}

Fruits of Vaccinium species are becoming increasingly popular due to their high levels of health-promoting bioactive compounds such as anthocyanins (Krikorian et al., 2010; Norberto et al., 2013; Chu et al., 2017; Shi et al., 2017; Zhou et al., 2020). In this study, the mechanism involved in anthocyanin accumulation in Wufanshu berries was investigated using UHPLC-MS/MS, RNA-Seq, dual-luciferase assays and transient color assays in tobacco leaves.

Anthocyanin composition and content has been reported to be highly variable among Vaccinium species and cultivars (Lohachoompol et al., 2008; You et al., 2011; Stevenson and Scalzo, 2012). Our results indicated that ripe Wufanshu berries are rich in a complex set of anthocyanins, including delphinidin, cyanidin, malvidin, peonidin, pelargonidin, and petunidin derivatives. The total anthocyanin content in Wufanshu berries is comparable to the highest content in blueberries as reported by other studies (Stevenson and Scalzo, 2012; Kim et al., 2013; Günther et al., 2020). Pelargonidin and its derivatives were shown to be absent in fruits of Vaccinium species such as highbush blueberry (Chung et al., 2016; Chai et al., 2021) and bilberry (Jaakola et al., 2002). However, four derivatives of pelargonidin (pelargonidin-3-O-galactoside, pelargonidin-3-O-glucoside, pelargonidin-3-O-rutinoside and pelargonidin-3,5-O-diglucoside) were detected in ripe Wufanshu berries (Supplementary Table 3). In future, it will be interesting to investigate anthocyanin composition of the berries of $V$. bracteatum species and elucidate the mechanisms and pathway enzymes responsible for the accumulation of pelargonidin and its derivatives.

Real-time quantitative RT-PCR analyses showed that whole pathway of anthocyanin biosynthetic genes was upregulated in Wufanshu berries during ripening. R2R3 MYB genes have been reported to act as anthocyanin activators in blueberry (Nguyen et al., 2017; Plunkett et al., 2018). Blast analysis enabled us to identify $V b M Y B A$, a homolog of the blueberry R2R3 MYB gene VcMYBA (Plunkett et al., 2018), from the transcriptome of Wufanshu berries. Sequence alignment and phylogenetic analysis suggested that the sub-group 6 VbMYBA is likely to be an anthocyanin activator. qRT-PCR analyses indicated that the expression of $V b M Y B A$ was well correlated with anthocyanin biosynthetic gene expression and anthocyanin accumulation in Wufanshu berries. Overexpression of $\mathrm{VbMYBA}$ with or without PsbHLH3 induced anthocyanin accumulation and red pigmentation in tobacco leaves. Dual-luciferase assays also show that VbMYBA can strongly activate the promoter of anthocyanin biosynthetic genes VcDFR and VavUFGT from other Vaccinium species. These results suggested that VbMYBA is an anthocyanin activator.

The anthocyanin-promoting ability of different MYB genes varied significantly (Zhou et al., 2018). Plunkett et al. (2018) show that transient overexpression of blueberry $V c M Y B A$ with $b H L H 3$ genes can induce a greater concentration of anthocyanin pigments in tobacco than apple MdMYB10 and peach PpMYB10.1. Zhou et al. (2018) demonstrated that two 
amino acid changes, Arg/Lys ${ }^{66}$ and Gly/Arg ${ }^{93}$, in the R3 repeat is responsible for anthocyanin-promoting activity divergence between peach $P p M Y B 10.1$ and PpMYB10.2. They further demonstrated that reciprocal substitution of $\mathrm{Arg} / \mathrm{Gly}^{93}$ between PpMYB10.1 and PpMYB10.2 affect their binding affinity to PpbHLH3 and suggested that bHLH-binding affinity is a key factor that determine the anthocyanin-promoting activity of MYB genes (Zhou et al., 2018). However, sequence alignment showed that the two amino acid and bHLH-binding domain were identical between VcMYBA and VbMYBA (Figure 3). The $\mathrm{N}$-terminal region of VbMYBA is longer than VcMYBA, but deletion of 23 amino acid-residue in the $\mathrm{N}$-terminal region of VbMYBA did not reduce its activity. In addition, ligation of the amino acid-residues to the N-terminal of VcMYBA did not enhance its anthocyanin promoting activity. These results suggest that the divergence of anthocyanin-promoting activity between VcMYBA and VbMYBA is likely to be due to the 19 different amino acids that distributed in $\mathrm{R}$ domains and C-terminal region. R2 and R3 domains of R2R3-MYBs play pivotal roles in interaction with DNA and determining their DNA binding affinity (Jia et al., 2004; Jiang et al., 2004). It has been reported that mutation of the $\mathrm{R} 2$ and $\mathrm{R} 3$ domains caused significant alteration in promoter target specificity and DNAbinding affinity of MYB proteins (Williams and Grotewold, 1997; Hernandez et al., 2004; Heppel et al., 2013). The C-terminus contains activation or repression and play an important role in determining the function of MYB proteins (Dubos et al., 2010; Feller et al., 2011). Variation of C-terminus also affect function of MYB proteins (Liu et al., 2016). Further study would be needed to identify the key amino acids responsible for the high anthocyanin-promoting activity of VbMYBA. It is also noteworthy that 23 amino acid-residue in the $\mathrm{N}$-terminal region of VbMYBA should be further verified at a protein level.

It has been demonstrated that bHLHs act as a crucial component in the regulation of anthocyanin biosynthesis. In Arabidopsis, the bHLH factors EGL3 and TT8 are necessary for anthocyanin accumulation in seedlings (Gonzalez et al., 2008). Several studies have indicated that bHLHs are essential for the anthocyanin activating activity of MYB proteins, such as potato R2R3 MYBs (Liu et al., 2016), peach PpMYB10.2 (Zhou et al., 2018), and apple MdMYB10 (Espley et al., 2007) in Nicotiana tabacum leaves. Infiltration of some MYBs, such as peach PpMYB10.1 (Zhou et al., 2015) and PpMYB10.4 (Zhou et al., 2014), Chinese bayberry MrMYB1 (Niu et al., 2010), litchi LcMYB1 (Lai et al., 2016), alone activated weak anthocyanin pigmentation in $N$. tabacum leaves, while co-infiltration with $b H L H$ s significantly enhanced anthocyanin accumulation. Kiwifruit AcMYB110 alone was able to induce strong anthocyanin accumulation in $N$. tabacum leaves (Peng et al., 2019). Similarly, infiltration of $V b M Y B A$ also

\section{REFERENCES}

Albert, N. W., Butelli, E., Moss, S. M. A., Piazza, P., Waite, C. N., Schwinn, K. E., et al. (2021). Discrete bHLH transcription factors play functionally overlapping roles in pigmentation patterning in flowers of Antirrhinum majus. New Phytol. 231, 849-863. doi: 10.1111/nph. 17142 triggered strong anthocyanin pigmentation in tobacco leaves and co-infiltration with PsbHLH3 has no significant effects on anthocyanin accumulation (Figures 5A,B and Supplementary Figure 1). One possibility is that $V b M Y B A$ can recruit bHLHs of $N$. tabacum to activate anthocyanin biosynthetic genes. Intriguingly, its homolog VcMYBA, which has a same bHLHbinding domain, cannot activate anthocyanin accumulation in N. tabacum leaves when infiltrated alone (Supplementary Figure 2). It will be interesting to investigate the underlying mechanisms that are responsible for MYBs, such as VbMYBA and AcMYB110, to activate anthocyanin accumulation without bHLHs in tobacco leaves.

\section{DATA AVAILABILITY STATEMENT}

The original contributions presented in the study are publicly available. This data can be found here: The raw transcriptome data have been deposited in the NCBI Sequence Read Archive under accession number PRJNA694726.

\section{AUTHOR CONTRIBUTIONS}

Z-ZF supervised the project. Y-LZ and Z-ZF wrote the manuscript. Y-LZ, Z-ZF, NA, CE, and CA participate in the experiments. KL-W, NA, CE, and RE provided scientific suggestion and revised the manuscript. All authors contributed to the article and approved the submitted version.

\section{FUNDING}

This research was funded by the Project of Fujian Academy of Agricultural Sciences (AA2018-16) and the China Scholarship Council (201909350001). Work at Plant \& Food Research was carried out under The New Zealand Ministry of Business, Innovation \& Employment (MBIE) contract C11 × 1704 "Filling the void: boosting the nutritional content of NZ fruit".

\section{ACKNOWLEDGMENTS}

We thank Monica Dragulescu and her team for plant care.

\section{SUPPLEMENTARY MATERIAL}

The Supplementary Material for this article can be found online at: https://www.frontiersin.org/articles/10.3389/fpls.2021. 697212/full\#supplementary-material

Albert, N. W., Davies, K. M., Lewis, D. H., Zhang, H., Montefiori, M., Brendolise, C., et al. (2014). A conserved network of transcriptional activators and repressors regulates anthocyanin pigmentation in eudicots. Plant Cell 26, $962-$ 980. doi: 10.1105/tpc.113.122069

Andre, C. M., Greenwood, J. M., Walker, E. G., Rassam, M., Sullivan, M., Evers, D., et al. (2012). Anti-inflammatory procyanidins and triterpenes in 109 apple varieties. J. Agric. Food Chem. 60, 10546-10554. doi: 10.1021/jf302809k 
Butelli, E., Garcia-Lor, A., Licciardello, C., Las Casas, G., Hill, L., Reforgiato Recupero, G., et al. (2017). Changes in anthocyanin production during domestication of Citrus. Plant Physiol. 173, 2225-2242. doi: 10.1104/pp.16. 01701

Chai, Z., Herrera-Balandrano, D. D., Yu, H., Beta, T., Zeng, Q., Zhang, X., et al. (2021). A comparative analysis on the anthocyanin composition of 74 blueberry cultivars from China. J. Food Comp. Anal. 102:104051. doi: 10.1016/j.jfca.2021. 104051

Chen, C., Chen, H., Zhang, Y., Thomas, H. R., Frank, M. H., He, Y., et al (2020). TBtools - an integrative toolkit developed for interactive analyses of big biological data. Mol. Plant 13, 1194-1202. doi: 10.1016/j.molp.2020.06.009

Cho, H.-S., Cho, Y.-S., and Cho, J.-A. J. A. H. (2012). Eco-physiological and horticultural characteristics of two Korean wild Vacciniums. Acta hortic. 926, $149-156$.

Chu, W., Gao, H., Cao, S., Fang, X., Chen, H., and Xiao, S. J. F. C (2017). Composition and morphology of cuticular wax in blueberry (Vaccinium spp.) fruits. Food Chem. 219, 436-442.

Chung, S. W., Yu, D. J., and Lee, H. J. (2016). Changes in anthocyanidin and anthocyanin pigments in highbush blueberry (Vaccinium corymbosum cv. Bluecrop) fruits during ripening. Hortic. Environ. Biotechnol. 57, 424-430. doi: 10.1007/s13580-016-0107-8

Die, J. V., Jones, R. W., Ogden, E. L., Ehlenfeldt, M. K., and Rowland, L. J. (2020). Characterization and analysis of anthocyanin-related genes in wild-type blueberry and the pink-fruited mutant cultivar 'Pink Lemonade': new insights into anthocyanin biosynthesis. Agronomy 10:1296.

Dubos, C., Stracke, R., Grotewold, E., Weisshaar, B., Martin, C., and Lepiniec, L. (2010). MYB transcription factors in Arabidopsis. Trends Plant Sci. 15, 573-581. doi: 10.1016/j.tplants.2010.06.005

Espley, R. V., Hellens, R. P., Putterill, J., Stevenson, D. E., Kutty-Amma, S., and Allan, A. C. (2007). Red colouration in apple fruit is due to the activity of the MYB transcription factor, MdMYB10. Plant J. 49, 414-427. doi: 10.1111/j.1365313X.2006.02964.x

Fan, M., Fan, Y., Huang, W., Wang, L., Li, Y., Qian, H., et al. (2018). Tentative characterization of precursor compounds and co-factors of pigment formation in production of 'wu mi' from Vaccinium bracteatum Thunb. Leaves. Food Chem. 262, 199-205. doi: 10.1016/j.foodchem.2018.04.101

Fan, M., Fan, Y., Rao, Z., Li, Y., Qian, H., Zhang, H., et al. (2019). Comparative investigation on metabolite changes in 'wu mi' production by Vaccinium bracteatum Thunb. leaves based on multivariate data analysis using UPLCQToF-MS. Food Chem. 286, 146-153. doi: 10.1016/j.foodchem.2019.01.144

Fan, M., Lian, W., Li, T., Fan, Y., Rao, Z., Li, Y., et al. (2020). Metabolomics approach reveals discriminatory metabolites associating with the blue pigments from Vaccinium bracteatum thunb. Leaves at different growth stages. Ind. Crops Prod. 147:112252. doi: 10.1016/j.indcrop.2020.112252

Fang, Z. Z., Zhou, D. R., Ye, X. F., Jiang, C. C., and Pan, S. L. (2016). Identification of candidate anthocyanin-related genes by transcriptomic analysis of 'Furongli' plum (Prunus salicina Lindl.) during fruit ripening using RNA-Seq. Front. Plant Sci. 7:1338. doi: 10.3389/fpls.2016.01338

Fang, Z.-Z., Lin-Wang, K., Zhou, D.-R., Lin, Y.-J., Jiang, C.-C., Pan, S.-L., et al. (2021). Activation of PsMYB10.2 transcription causes anthocyanin accumulation in flesh of the red-fleshed mutant of 'Sanyueli' (Prunus salicina Lindl.). Front. Plant Sci. 12:1167. doi: 10.3389/fpls.2021.680469

Feller, A., Machemer, K., Braun, E. L., and Grotewold, E. (2011). Evolutionary and comparative analysis of MYB and bHLH plant transcription factors. Plant J. 66, 94-116. doi: 10.1111/j.1365-313X.2010.04459.x

Feng, S., Sun, S., Chen, X., Wu, S., Wang, D., and Chen, X. (2015). PyMYB10 and PyMYB10.1 interact with bHLH to enhance anthocyanin accumulation in pears. PLoS One 10:e0142112. doi: 10.1371/journal.pone.0142112

Gonzalez, A., Zhao, M., Leavitt, J. M., and Lloyd, A. M. (2008). Regulation of the anthocyanin biosynthetic pathway by the TTG1/bHLH/Myb transcriptional complex in Arabidopsis seedlings. Plant J. 53, 814-827. doi: 10.1111/j.1365313X.2007.03373.x

Grabherr, M. G., Haas, B. J., Yassour, M., Levin, J. Z., Thompson, D. A., and Amit, I. (2011). Full-length transcriptome assembly from RNA-Seq data without a references genome. Nat. Biotechnol. 29, 644-652. doi: 10.1038/nbt.1883

Günther, C. S., Dare, A. P., McGhie, T. K., Deng, C., Lafferty, D. J., Plunkett, B. J., et al. (2020). Spatiotemporal modulation of flavonoid metabolism in blueberries. Front. Plant Sci. 11:545. doi: 10.3389/fpls.2020.00545

Hellens, R. P., Allan, A. C., Friel, E. N., Bolitho, K., Grafton, K., Templeton, M. D., et al. (2005). Transient expression vectors for functional genomics, quantification of promoter activity and RNA silencing in plants. Plant Methods 1:13. doi: 10.1186/1746-4811-1-13

Heppel, S. C., Jaffe, F. W., Takos, A. M., Schellmann, S., Rausch, T., Walker, A. R., et al. (2013). Identification of key amino acids for the evolution of promoter target specificity of anthocyanin and proanthocyanidin regulating MYB factors. Plant Mol. Biol. 82, 457-471. doi: 10.1007/s11103-013-0074-8

Hernandez, J. M., Heine, G. F., Irani, N. G., Feller, A., Kim, M.-G., Matulnik, T., et al. (2004). Different mechanisms participate in the R-dependent activity of the R2R3 MYB transcription factor C1. J. Biol. Chem. 279, 48205-48213. doi: 10.1074/jbc.M407845200

Hichri, I., Barrieu, F., Bogs, J., Kappel, C., Delrot, S., and Lauvergeat, V. (2011). Recent advances in the transcriptional regulation of the flavonoid biosynthetic pathway. J. Exp. Bot. 62, 2465-2483. doi: 10.1093/jxb/erq442

Holton, T. A., and Cornish, E. C. (1995). Genetics and biochemistry of anthocyanin aiosynthesis. Plant Cell 7, 1071-1083. doi: 10.1105/tpc.7.7.1071

Jaakola, L. (2013). New insights into the regulation of anthocyanin biosynthesis in fruits. Trends Plant Sci. 18, 477-483. doi: 10.1016/j.tplants.2013.06.003

Jaakola, L., Määttä, K., Pirttilä, A. M., Törrönen, R., Kärenlampi, S., and Hohtola, A. (2002). Expression of genes involved in anthocyanin biosynthesis in relation to anthocyanin, proanthocyanidin, and flavonol levels during bilberry fruit development. Plant Physiol. 130, 729-739.

Jia, L., Clegg, M. T., and Jiang, T. (2004). Evolutionary dynamics of the DNAbinding domains in putative R2R3-MYB genes identified from rice subspecies indica and japonica genomes. Plant Physiol. 134, 575-585. doi: 10.1104/pp.103. 027201

Jiang, C., Gu, X., and Peterson, T. (2004). Identification of conserved gene structures and carboxy-terminal motifs in the Myb gene family of Arabidopsis and Oryza sativa L. ssp. indica. Genome Biol. 5:R46. doi: 10.1186/gb-20045-7-r46

Jin, W., Wang, H., Li, M., Wang, J., Yang, Y., Zhang, X., et al. (2016). The R2R3 MYB transcription factor PavMYB10.1 involves in anthocyanin biosynthesis and determines fruit skin colour in sweet cherry (Prunus avium L.). Plant Biotechnol. J. 14, 2120-2133. doi: 10.1111/pbi.12568

Kim, J. G., Kim, H. L., Kim, S. J., and Park, K.-S. (2013). Fruit quality, anthocyanin and total phenolic contents, and antioxidant activities of 45 blueberry cultivars grown in Suwon, Korea. J. Zhejiang Univ. Sci. B 14, 793-799. doi: 10.1631/jzus. B1300012

Kitamura, S., Akita, Y., Ishizaka, H., Narumi, I., and Tanaka, A. (2012). Molecular characterization of an anthocyanin-related glutathione S-transferase gene in cyclamen. J. Plant Physiol. 169, 636-642. doi: 10.1016/j.jplph.2011.12.011

Kobayashi, S., Ishimaru, M., Hiraoka, K., and Honda, C. (2002). Myb-related genes of the Kyoho grape (Vitis labruscana) regulate anthocyanin biosynthesis. Planta 215, 924-933. doi: 10.1007/s00425-002-0830-5

Krikorian, R., Shidler, M. D., Nash, T. A., Kalt, W., Vinqvist-Tymchuk, M. R., Shukitt-Hale, B., et al. (2010). Blueberry supplementation improves memory in older adults. J. Agric. Food Chem. 58, 3996-4000. doi: 10.1021/jf9029332

Lai, B., Du, L., Liu, R., Hu, B., Su, W., Qin, Y., et al. (2016). Two LcbHLH transcription factors interacting with $L c M Y B 1$ in regulating late structural genes of anthocyanin biosynthesis in Nicotiana and Litchi chinensis during anthocyanin accumulation. Front. Plant Sci. 7:166. doi: 10.3389/fpls.2016. 00166

Landa, P., Skalova, L., Bousova, I., Kutil, Z., Langhansova, L., Lou, J.-D., et al. (2014). In vitro anti-proliferative and anti-inflammatory activity of leaf and fruit extracts from Vaccinium bracteatum Thunb. Pak. J. Pharm. Sci. 27, 103-106.

Langmead, B., Trapnell, C., Pop, M., and Salzberg, S. L. (2009). Ultrafast and memory-efficient alignment of short DNA sequences to the human genome. Genome Biol. 10, 1-10. doi: 10.1186/gb-2009-10-3-r25

Lee, S., Jung, E. S., Do, S.-G., Jung, G.-Y., Song, G., Song, J.-M., et al. (2014). Correlation between species-specific metabolite profiles and bioactivities of blueberries (Vaccinium spp.). J. Agric. Food Chem. 62, 2126-2133. doi: 10.1021/ jf405272b

Li, B., and Dewey, C. N. (2011). RSEM: accurate transcript quantification from RNA-Seq data with or without a reference genome. BMC Bioinformatics 12:323. doi: 10.1186/1471-2105-12-323

Li, D., Li, B., Ma, Y., Sun, X., Lin, Y., and Meng, X. (2017). Polyphenols, anthocyanins, and flavonoids contents and the antioxidant capacity of various cultivars of highbush and half-high blueberries. J. Food Comp. Anal. 62, 84-93. doi: 10.1016/j.jfca.2017.03.006

Lin-Wang, K., Bolitho, K., Grafton, K., Kortstee, A., Karunairetnam, S., McGhie, T., et al. (2010). An R2R3 MYB transcription factor associated with regulation 
of the anthocyanin biosynthetic pathway in Rosaceae. BMC Plant Biol. 10:50. doi: 10.1186/1471-2229-10-50

Liu, Y., Lin-Wang, K., Espley, R. V., Wang, L., Yang, H., Yu, B., et al. (2016). Functional diversification of the potato R2R3 MYB anthocyanin activators AN1, MYBA1, and MYB113 and their interaction with basic helix-loop-helix cofactors. J. Exp. Bot. 67, 2159-2176. doi: 10.1093/jxb/erw014

Lohachoompol, V., Mulholland, M., Srzednicki, G., and Craske, J. (2008). Determination of anthocyanins in various cultivars of highbush and rabbiteye blueberries. Food Chem. 111, 249-254. doi: 10.1016/j.foodchem.2008.03.067

Montefiori, M., Brendolise, C., Dare, A. P., Lin-Wang, K., Davies, K. M., Hellens, R. P., et al. (2015). In the Solanaceae, a hierarchy of bHLHs confer distinct target specificity to the anthocyanin regulatory complex. J. Exp. Bot. 66, 1427-1436. doi: $10.1093 / \mathrm{jxb} / \mathrm{eru} 494$

Nesi, N., Jond, C., Debeaujon, I., Caboche, M., and Lepiniec, L. (2001). The Arabidopsis TT2 gene encodes an R2R3 MYB domain protein that acts as a key determinant for proanthocyanidin accumulation in developing seed. Plant Cell 13, 2099-2114.

Nguyen, C. T. T., Lim, S., Lee, J. G., and Lee, E. J. (2017). VcBBX, VcMYB21, and VCR2R3MYB transcription factors are involved in UV-B-induced anthocyanin biosynthesis in the peel of harvested blueberry fruit. J. Agric. Food Chem. 65, 2066-2073. doi: 10.1021/acs.jafc.6b05253

Niu, S., Xu, C., Zhang, W., Zhang, B., Li, X., Lin-Wang, K., et al. (2010). Coordinated regulation of anthocyanin biosynthesis in Chinese bayberry (Myrica rubra) fruit by a R2R3 MYB transcription factor. Planta 231, 887-899. doi: $10.1007 / \mathrm{s} 00425-009-1095-\mathrm{z}$

Norberto, S., Silva, S., Meireles, M., Faria, A., Pintado, M., and Calhau, C. (2013). Blueberry anthocyanins in health promotion: a metabolic overview. J. Funct. Foods 5, 1518-1528. doi: 10.1016/j.jff.2013.08.015

Oh, D. R., Kim, Y., Choi, E. J., Jung, M. A., Oh, K. N., Hong, J. A., et al. (2018a). Antidepressant-Like effects of Vaccinium bracteatum in chronic restraint stress mice: functional actions and mechanism explorations. Am. J. Chin. Med. 46, 357-387. doi: $10.1142 / \mathrm{s} 0192415 \times 18500180$

Oh, D.-R., Yoo, J.-S., Kim, Y., Kang, H., Lee, H., Lm, S. J., et al. (2018b). Vaccinium bracteatum leaf extract reverses chronic restraint stress-induced depression-like behavior in mice: regulation of hypothalamic-pituitary-adrenal axis, serotonin turnover systems, and ERK/Akt phosphorylation. Front. Pharmacol. 9:604. doi: 10.3389/fphar.2018.00604

Peng, Y., Lin-Wang, K., Cooney, J. M., Wang, T., Espley, R. V., and Allan, A. C. (2019). Differential regulation of the anthocyanin profile in purple kiwifruit (Actinidia species). Hortic. Res. 6:3. doi: 10.1038/s41438-018-0076-4

Plunkett, B. J., Espley, R. V., Dare, A. P., Warren, B. A. W., Grierson, E. R. P., Cordiner, S., et al. (2018). MYBA from blueberry (Vaccinium Section Cyanococcus) is a subgroup 6 type R2R3MYB transcription factor that activates anthocyanin production. Front. Plant Sci. 9:1300. doi: 10.3389/fpls.2018.01300

Primetta, A. K., Karppinen, K., Riihinen, K. R., and Jaakola, L. (2015). Metabolic and molecular analyses of white mutant Vaccinium berries show downregulation of MYBPA1-type R2R3 MYB regulatory factor. Planta 242, 631-643. doi: $10.1007 / \mathrm{s} 00425-015-2363-8$

Robert, X., and Gouet, P. (2014). Deciphering key features in protein structures with the new ENDscript server. Nucleic Acids Res. 42, W320-W324.

Shi, M., Loftus, H., McAinch, A. J., and Su, X. Q. (2017). Blueberry as a source of bioactive compounds for the treatment of obesity, type 2 diabetes and chronic inflammation. J. Funct. Foods 30, 16-29. doi: 10.1016/j.jff.2016.12.036

Stevenson, D., and Scalzo, J. J. J. O. B. R (2012). Anthocyanin composition and content of blueberries from around the world. J. Berry Res. 2, 179-189.

Tuan, P. A., Bai, S., Yaegaki, H., Tamura, T., Hihara, S., Moriguchi, T., et al. (2015). The crucial role of PpMYB10.1 in anthocyanin accumulation in peach and relationships between its allelic type and skin color phenotype. BMC Plant Biol. 15:280. doi: 10.1186/s12870-015-0664-5

Wang, L., Dong, M., and Yao, H. (2004). The nutritive value of black berry fruit of Vaccinium bracteatum Thunb. and its exploitation and utilization. Zhong Cao Yao 35, 14-15.

Wang, L., Zhang, X. T., Zhang, H. Y., Yao, H. Y., and Zhang, H. (2010). Effect of Vaccinium bracteatum Thunb. leaves extract on blood glucose and plasma lipid levels in streptozotocin-induced diabetic mice. J. Ethnopharmacol. 130, 465-469. doi: 10.1016/j.jep.2010.05.031

Wang, L., Zhang, Y., Xu, M., Wang, Y., Cheng, S., Liebrecht, A., et al. (2013). Anti-diabetic activity of Vaccinium bracteatum Thunb. leaves' polysaccharide in STZ-induced diabetic mice. Int. J. Biol. Macromol. 61, 317-321. doi: 10.1016/ j.ijbiomac.2013.07.028

Williams, C. E., and Grotewold, E. (1997). Differences between plant and animal Myb domains are fundamental for DNA binding activity, and chimeric Myb domains have novel DNA binding specificities. J. Biol. Chem. 272, 563-571. doi: $10.1074 / j b c .272 .1 .563$

Xu, Y., Fan, M., Zhou, S., Wang, L., Qian, H., Zhang, H., et al. (2017). Effect of Vaccinium bracteatum Thunb. leaf pigment on the thermal, pasting, and textural properties and microstructure characterization of rice starch. Food Chem. 228, 435-440. doi: 10.1016/j.foodchem.2017.02.041

Yang, Y., Cui, B., Tan, Z., Song, B., Cao, H., and Zong, C. (2018). RNA sequencing and anthocyanin synthesis-related genes expression analyses in white-fruited Vaccinium uliginosum. BMC Genomics 19:930. doi: 10.1186/s12864-0185351-0

You, Q., Wang, B., Chen, F., Huang, Z., Wang, X., and Luo, P. G. (2011). Comparison of anthocyanins and phenolics in organically and conventionally grown blueberries in selected cultivars. Food Chem. 125, 201-208. doi: 10.1016/ j.foodchem.2010.08.063

Zhang, J., Chu, C.-J., Li, X.-L., Yao, S., Yan, B., Ren, H.-L., et al. (2014). Isolation and identification of antioxidant compounds in Vaccinium bracteatum Thunb. by UHPLC-Q-TOF LC/MS and their kidney damage protection. J. Funct. Foods 11, 62-70. doi: 10.1016/j.jff.2014.09.005

Zhang, Y., Fang, Z., Ye, X., and Pan, S. (2018). Identification of candidate genes involved in anthocyanin accumulation in the peel of jaboticaba (Myrciaria cauliflora) fruits by transcriptomic analysis. Gene 676, 202-213. doi: 10.1016/ j.gene.2018.07.039

Zheng, Y., Chen, L., Liu, Y., Shi, L., Wan, S., and Wang, L. (2019). Evaluation of antimicrobial activity of water-soluble flavonoids extract from Vaccinium bracteatum Thunb. leaves. Food Sci. Biotechnol. 28, 1853-1859. doi: 10.1007/ s10068-019-00634-4

Zhou, H., Liao, L., Xu, S., Ren, F., Zhao, J., Ogutu, C., et al. (2018). Two amino acid changes in the R3 repeat cause functional divergence of two clustered MYB10 genes in peach. Plant Mol. Biol. 98, 169-183. doi: 10.1007/s11103-0180773-2

Zhou, H., Lin-Wang, K., Wang, H., Gu, C., Dare, A. P., Espley, R. V., et al. (2015). Molecular genetics of blood-fleshed peach reveals activation of anthocyanin biosynthesis by NAC transcription factors. Plant J. 82, 105-121. doi: 10.1111/ tpj.12792

Zhou, L., Xie, M., Yang, F., and Liu, J. (2020). Antioxidant activity of high purity blueberry anthocyanins and the effects on human intestinal microbiota. $L W T$ 117:108621. doi: 10.1016/j.lwt.2019.108621

Zhou, Y., Zhou, H., Lin-Wang, K., Vimolmangkang, S., Espley, R. V., Wang, L., et al. (2014). Transcriptome analysis and transient transformation suggest an ancient duplicated MYB transcription factor as a candidate gene for leaf red coloration in peach. BMC Plant Biol. 14:1-13. doi: 10.1186/s12870-0140388-y

Zifkin, M., Jin, A., Ozga, J. A., Zaharia, L. I., Schernthaner, J. P., Gesell, A., et al. (2011). Gene expression and metabolite profiling of developing highbush blueberry fruit indicates transcriptional regulation of flavonoid metabolism and activation of abscisic acid metabolism. Plant Physiol. 158, 200-224.

Conflict of Interest: The authors declare that the research was conducted in the absence of any commercial or financial relationships that could be construed as a potential conflict of interest.

Publisher's Note: All claims expressed in this article are solely those of the authors and do not necessarily represent those of their affiliated organizations, or those of the publisher, the editors and the reviewers. Any product that may be evaluated in this article, or claim that may be made by its manufacturer, is not guaranteed or endorsed by the publisher.

Copyright (๑) 2021 Zhang, Lin-Wang, Albert, Elborough, Espley, Andre and Fang. This is an open-access article distributed under the terms of the Creative Commons Attribution License (CC BY). The use, distribution or reproduction in other forums is permitted, provided the original author(s) and the copyright owner(s) are credited and that the original publication in this journal is cited, in accordance with accepted academic practice. No use, distribution or reproduction is permitted which does not comply with these terms. 